\title{
Response to: Interval appendicectomy in children clinical outcomes, financial costs and patient benefits. David Fawkner- Corbett, Wajid J Jawaid, Jo McPartland, Paul D Losty Pediatr Surg Int (2014) 30:743-746
}

\author{
Raj Lahiri $\cdot$ Caroline Pardy $\cdot$ Anies Mahomed
}

Accepted: 15 August 2014/Published online: 27 August 2014

(c) Springer-Verlag Berlin Heidelberg 2014

\section{Dear Sir,}

The authors of the above retrospective review of 61 children with appendix mass coming to interval appendicectomy (IA) conclude that this may be 'non-essential surgery' [1]. Their contention is based on the number of cases developing recurrent appendicitis (12\%), in the period (median 76 days) awaiting interval surgery, and the costs incurred ( $£ 1936$ per case) in delivering this option. The latter was calculated on a median stay of 2-3 days depending on whether a laparoscopic or conventional technique, respectively, was employed.

However, in an era of pervasive minimally invasive surgery, in experienced hands, the arguments in support of an expectant approach to IA are being challenged. In a personal series ${ }^{1}$ of 15 cases of appendix mass, prospectively recorded over an 8-year period (2005-14), with a mean interval to surgery of 122 days, five cases (33\%) required re-admission and antibiotics while awaiting IA. Utilising exclusively a laparoscopic approach at IA there were no conversions. Operative time was $45.8 \mathrm{~min}$ (SD $12.35)$, with the majority performed as day cases $(n=8)$ and the rest staying overnight. There were no intra-operative complications. Two cases had minor problems ( 1 chest pain, 1 vomiting) requiring brief re-admission without need

This comment refers to the article available at doi:10.1007/s00383014-3521-y.

R. Lahiri · C. Pardy

The Royal Alexandra Children's Hospital, Brighton, UK

e-mail: carolinepardy@gmail.com

A. Mahomed ( $\square)$

Department of Paediatric Surgery, Royal Alexandra Children's

Hospital, Eastern Road, Brighton BN2 5BE, UK

e-mail: Anies.Mahomed@bsuh.nhs.uk for further surgery. The theatre cost per case based on a standard inventory and consumable usage was £212 [2, 3], and the overall costs factoring for both in hospital stay and drug usage is likely to be very competitive relative to that quoted above.

The weakness in the expectant approach to appendix mass is the unpredictability of anticipating the risk and timing of further attacks of appendicitis, and consequently the difficulty in reassuring parents about the future health of their child. Histology, in our series, revealed active inflammation in 12 cases with another demonstrating a faecolith within the appendix. Although not all of these cases would have progressed to clinically significant disease, the risk of further problems is real. Given this scenario, the option of IA delivered laparoscopically, with minimal in-hospital stay and cost implication, is an attractive and rational choice. Unfortunately, the on-going CHINA trial will not specifically be examining the impact of the laparoscopic approach per se in the overall performance of these cases [4].

\section{References}

1. Fawkner-Corbett D, Jawaid WJ, McPartland J, Losty PD (2014) Interval appendicectomy in children clinical outcomes, financial costs and patient benefits. Pediatr Surg Int 30:743-746

2. Mahomed AA, McLean V (2007) Cost analysis of minimally invasive surgery in a paediatric setting. J Laparoendosc Adv Surg Tech A 17(3):375-379

3. Mahomed AA, Fartacek R, MacLean V (2005) Appendicectomy: keeping it safe, simple and cost effective. Laparoendosc Adv Surg Tech A 15(2):221

4. ISRCTN. http://www.chinastudy.org.uk-Trial. Registration no. SRCN 93815412

\footnotetext{
${ }_{1}$ Anies Mahomed, Department of Paediatric Surgery, Royal Alexandra Children's Hospital, Eastern Road, Brighton, BN2 5BE.
} 